\title{
Sequence variants of the HTR3A gene contribute to the genetic prediction of postoperative nausea in Taiwan
}

\author{
Yi-Mei Joy Lin ${ }^{1}$, Cheng-Da Hsu², Hsiao-Yen Hsieh², Chia-Chih Alex Tseng ${ }^{3}$ and H Sunny Sun ${ }^{4}$ \\ Postoperative nausea (PON) is a common complication, and therefore, it is important to identify the associated genetic factors \\ and the candidate predictive markers. Current clinical and basic research suggests that the 5-hydroxytryptamine type 3A receptor \\ (HTR3A) may be important in the occurrence of PON. The association between three single nucleotide polymorphisms (SNPs) of \\ the HTR3A gene and PON was examined to determine whether this can be used to predict the incidence of PON in a unique \\ Taiwanese population without any reported postoperative nausea and vomiting (PONV) risk factors associated with PON \\ occurrence. One thousand adult surgical patients who received general anesthesia were included in this analysis. A total of 369 \\ patients were finally selected for a two-stage association study. Significant single-locus associations for all three HTR3A SNPs \\ and PON were identified in both stages. In addition, two of the most common haplotypes, CTT and TAG, showed both a \\ significant risk for and a protective effect against PON, respectively. Our findings support the notion that different haplotypes \\ of HTR3A have reciprocal effects in the etiology of PON. Therefore specific haplotypes of HTR3A may be useful as predictors \\ of PON for $24 \mathrm{~h}$ immediately after surgery in our population.
}

Journal of Human Genetics (2014) 59, 655-660; doi:10.1038/jhg.2014.89; published online 23 October 2014

\section{INTRODUCTION}

Postoperative nausea (PON) is an important component of postoperative nausea and vomiting (PONV), and it is one of the most common complications following anesthesia. The general incidence of PONV is around $20-30 \%$ but may be as high as $80 \%$ in high-risk patients. ${ }^{1-3}$ Many scoring systems have been developed to identify clinical risk parameters and to establish PONV prediction models, ${ }^{4-6}$ but most of them have moderate discriminating power and, therefore, are limited as clinically accurate or useful. Apparently, some important risk factors have been overlooked, including possible genetic factors. However, few studies have focused on identifying the genetic factors associated with PONV. Additionally, our population is unique, because no significant associations between reported PONV risk factors and PON occurrence have been found. Thus it is important to evaluate the possible genetic factors that can be used to predict nausea after general anesthesiology in this unique population.

Physiologically, PONV is characterized by two distinct but related phenomena: nausea and vomiting. However, the incidence rate of PON is usually higher than that of postoperative vomiting (POV) ${ }^{7,8}$ The risk factors involved might be different. ${ }^{9}$ Unlike POV, with its quantifiable objective events, PON is a subjective feeling that can be accompanied by perspiration, salivation, tachycardia, anorexia and headache. Thus the mechanisms that lead to nausea are less clear than those that lead to vomiting, ${ }^{10,11}$ and it is important to clarify the factors of PON.

A family history of PON has been considered an important risk factor and suggests that the genetic background might be a significant modulator of the occurrence and phenotypic variability of PON. ${ }^{12,13}$ Risk-assessment knowledge based on the relative impact of independent risk factors will help physicians stratify patients into groups that require different clinical management. Current research suggests that the 5-hydroxytryptamine type 3 receptors (HTR3s) are important in the occurrence of PON. ${ }^{14}$ It is proposed that HTR3 sends signals to a chemoreceptor trigger zone in the medulla oblongata under stimulation from nausea-inducing agents. HTR3 antagonists have been widely used to prevent and treat PON and selective serotonin reuptake inhibitor-induced nausea. ${ }^{15-17}$

As a ligand-gated ion channel, a functional HTR3 receptor channel is composed of five subunits. The essential subunit of the functional HTR3 channel is HTR3A, which is also the only component that can form functional homopentameric HTR3 channels. ${ }^{18,19}$ Although the HTR3A gene has attracted interest because of its effects on $\mathrm{PON},{ }^{16,17,20,21}$ there is no published study of a complete assessment of the association of HTR3A genetic background with PON. We, therefore, hypothesize that genetic variations in HTR3A genes modulate the susceptibility to PON.

${ }^{1}$ Institute of Biomedical Sciences, National Chung Hsing University, Taichung, Taiwan; ${ }^{2}$ Department of Medical Research, Ditmanson Medical Foundation Chia-Yi Christian Hospital, Chia-Yi, Taiwan; ${ }^{3}$ Department of Anesthesiology, Ditmanson Medical Foundation Chia-Yi Christian Hospital, Chia-Yi, Taiwan and ${ }^{4}$ Institute of Basic Medical Sciences and Institute of Molecular Medicine, National Cheng Kung University Medical College, Tainan, Taiwan

Correspondence: Professor C-CA Tseng, Department of Anesthesiology, Ditmanson Medical Foundation Chia-Yi Christian Hospital, 539 Chunghsiao Road, Chia-Yi 60002, Taiwan. E-mail: cctmay888@yahoo.com.tw

or Professor HS Sun, Institute of Molecular Medicine, National Cheng Kung University Medical College, 1 University Road, Tainan 70101, Taiwan.

E-mail: hssun@mail.ncku.edu.tw

Received 27 March 2014; revised 27 August 2014; accepted 11 September 2014; published online 23 October 2014 
To clarify the impact of HTR3A polymorphisms on risks of PON after general anesthesia, we applied a powerful two-stage association study of 369 postoperative patients who underwent 9 different types of surgery in a Taiwanese population. Our analysis reveals significant associations between PON symptoms and HTR3A polymorphisms on both single-locus and haplotype levels in two-stage studies. The results suggest that the HTR $3 A$ gene may have a role in the pathogenesis of PON, and the polymorphisms of HTR $3 A$ may be candidate predictors of PON during the $24 \mathrm{~h}$ immediately following surgery in our population.

\section{METHODS}

\section{Ethics statement}

The Ethics Committee of the Ditmanson Medical Foundation Chia-Yi Christian Hospital approved this study. All participants provided written informed consent.

\section{Study participants and DNA preparation}

Adult patients ( $>20$ years old) scheduled to undergo general anesthesia gave their informed written consent to join this prospective perioperative outcome cohort. Patients undergoing emergency surgery, those who could not communicate well and those who refused to participate in our study were excluded. A total of 369 cases were extracted from our 1000 case cohort that did not receive anti-emesis (Dexamethasone or Droperidol) and had general anesthesia. Genomic DNA was prepared from peripheral blood using the DNA extraction kits (QuickGene DNA Whole Blood Kit; Fujifilm Life Science, Stanford, CT, USA).

The patients' demographic data — gender, age, body weight, height, calculated BMI, smoking status and other validated PONV risks-were recorded by one of the two research assistants (RAs) during preoperative visits to the ward. No preoperative medicine like sedation, opioids or anti-emesis was given to any of the patients. Before the induction of anesthesia, standard monitoring (non-invasive arterial pressure measurement, electrocardiography with heart rate and arterial oxygen saturation (pulse oximeter)) was set to routine. After preoxygenation, anesthesia was administered under the anesthesiologist's order. The anesthesiologists used standard tracheal intubation. All patients underwent volume-controlled ventilation with a tidal volume of $7-10 \mathrm{ml} \mathrm{kg}^{-1}$ and a respiratory rate of 10-12 breaths per minute without nitrous oxide. During the surgical procedure, the RA documented the patient's course and anesthetic used. Recording of specific anesthetic drugs was focused on what volatile was used and the types and dosages of opioids that were used irrespective of the specific compound. The data also included the surgical procedures and preventive and rescue anti-emetic medications. Patients who received antiemesis were excluded from analysis in order to control the confounding factor.

\section{Phenotype scoring method}

The main outcome of interest was any incidence of PON in the postoperation period of $24 \mathrm{~h}$. All the PON incidences with the patients were scored by one of the RAs in the postanesthesia recovery room (PAR) $24 \mathrm{~h}$ after operation. Any incidence of nausea that was recorded either in the PAR or 2-24h after operation was presented as yes with or no without PON as the phenotype outcome.

\section{Study design}

Selected single nucleotide polymorphisms (SNPs) of the HTR3A gene were genotyped and tested as risk factors of a PON phenotype. Occurrences of nausea during the PAR period or $24 \mathrm{~h}$ after operation were recorded; associations between HTR3A genotypes and PON occurrence were analyzed. To test whether the HTR3A genotype had different effects on PON after different types of surgeries, participants were divided into groups and evaluated using a two-stage association analysis (Figure 1). First, we did genetic exploratory analyses on 171 patients who underwent lower (operation 1) and upper (operation 2) abdominal surgery, which involves an opened abdominal cavity. A second genetic extension study included an additional 198 patients who received operations 3-9 (total 369 patients) recruited after undergoing various types of surgeries. There were nine different types of surgeries, as follows: lower abdominal (Operation 1; Op. 1; 114 patients), upper abdominal

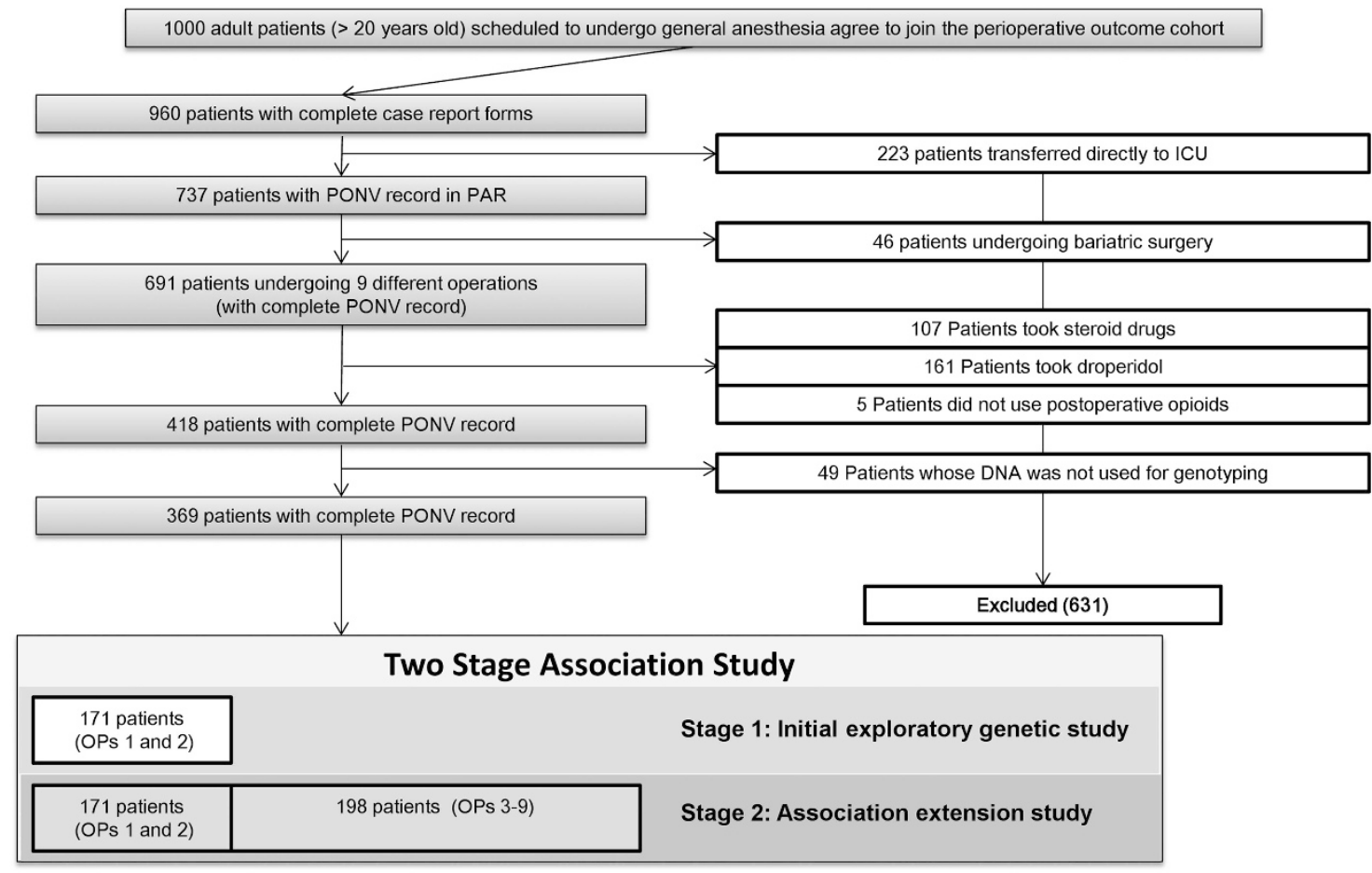

Figure 1 Flow chart showing the study design and patient recruitment. Three hundred sixty-nine patients who underwent general anesthesia were recruited. The flow chart shows the study design and the steps involved in patient recruitment. The two rows in the Two-Stage Association Study section show patients and operation types for each stage of the study. ICU, Intensive Care Unit; OP, operation; PAR, postanesthesia recovery room. 
Table 1 Human HTR3A polymorphisms genotyped in this study

\begin{tabular}{|c|c|c|c|c|c|}
\hline \multirow[b]{2}{*}{$d b S N P I D$} & \multirow[b]{2}{*}{ Region in HTR3A } & \multirow[b]{2}{*}{ TaqMan Assay ID } & \multirow[b]{2}{*}{ Context sequence $[\mathrm{VIC} / \mathrm{FAM}]^{\mathrm{a}}$} & \multicolumn{2}{|c|}{ Alleles } \\
\hline & & & & $D N A$ & Amino acid \\
\hline rs33940208 & Coding sequence & C-22275584 & AGCAGGCGCTGCT[C/T]GCCTTGCTCCTC & $\mathrm{C} / \mathrm{T}$ & Leu16Leu \\
\hline rs1985242 & Intron 1 & C-1372137 & TGCTTTCCTGTCA[A/T]TGAATGCATAGG & $T / A$ & NA \\
\hline rs10160548 & Intron 5 & C-1372126 & CCATCATCACAGG[G/T]TCCAGCAGGCTC & $\mathrm{T} / \mathrm{G}$ & NA \\
\hline
\end{tabular}

Abbreviations: HTR3A, 5-hydroxytryptamine type 3 A receptor; NA, not applicable; dbSNP, Single-Nucleotide Polymorphism database.

${ }^{a}$ Bold characters indicate the alleles of targeted SNPs.

(Op. 2; 57 patients), thoracic (Op. 3; 45 patients), cardiac (Op. 4; 6 patients), spinal (Op. 5; 9 patients), laparoscopic intra-abdominal (Op. 6; 30 patients), thyroid (Op. 7; 79 patients), breast (Op. 8; 24 patients), and oral (Op. 9; 5 patients).

\section{Marker selection and genotyping procedures}

Three SNPs within the human HTR3A locus: rs33940208, rs1985242, and rs10160548 were selected from the Single-Nucleotide Polymorphism database (dbSNP) (http://www.ncbi.nlm.nih.gov/SNP) for the study, depending on their relative functional significance, relative genomic distribution and allele frequencies in our population. SNPs with a minor allele frequency of $<0.05$ and frequencies between two alternative alleles are too close to each other were excluded. ${ }^{22}$ Briefly, SNPs in the coding was the most important candidate; and two intronic polymorphisms with minor allele frequency are between $10 \%$ and $40 \%$ were also selected to be uniformly distributed across the HTR3A gene and provide more information in an association study. We used the TaqMan SNP Genotyping Assay (Applied Biosystems, Foster City, CA, USA) to genotype all participants in this study. After the design of primers and TaqMan probes, genotyping was done using a sequence detection system (ABI PRISM 7500; Applied Biosystems). Detailed information, assay IDs and context sequences for each selected marker are listed in Table 1.

\section{Statistical analysis}

We determined the phenotype as the incidence of PON in the postoperative $24 \mathrm{~h}$. Differences in demographic data and risk factor distributions between PON patients and non-nausea patients were analyzed using a $\chi^{2}$ test by SPSS (SPSS Inc., Chicago, IL, USA). For the two-stage association study, the homogeneity of odds ratio (OR) for the two data sets were tested using the Breslow-Day statistic, and the common OR and overall degree of association were estimated using Mantel-Haenszel method by SPSS. The Hardy-Weinberg equilibrium of each marker and differences in genotype distributions between PON patients and non-nausea patients was analyzed using a $\chi^{2}$ test (SNPAlyze 4.1; Dynacom Co, Kanagawa, Japan). Standardized pairwise disequilibrium coefficients $\left(D^{\prime}\right)$ for measuring linkage disequilibrium (LD) between the three SNPs, the construction of haplotypes and haplotype-based association were also estimated using SNPAlyze.

\section{RESULTS}

From 2009 to 2011, we invited 1000 eligible patients to participate in the study. In all, 960 surgical patients with complete case report forms of PON were included in this analysis. In addition to 223 patients transferred directly to the Intensive Care Unit, there were 737 with PON data in the PAR or during the postoperative $24 \mathrm{~h}$. Patients who took prophylactic drugs, such as dexamethasone or droperidol, and patients who did not take postoperative opioids were excluded to remove the possible influence of those drugs on PON outcomes. Patients under bariatric surgery was also excluded from the study for high percentage of PONV (80\%) observed in this cohort. Finally, 369 patients were involved in this study. A flow chart of the study design and the selection of the final set of recruited patients is shown in Figure 1.
Table 2 Demographic data of individuals collected in this study

\begin{tabular}{lccc}
\hline & Nausea & Non-nausea & P-value $^{\text {a }}$ \\
\hline Gender (F/M) & $53 / 29$ & $187 / 135$ & 0.170 \\
Smoking status (N/Y) & $58 / 24$ & $205 / 117$ & 0.142 \\
Alcohol drinking (N/Y) & $71 / 10$ & $255 / 66$ & 0.059 \\
Car sick (N/Y) & $49 / 31$ & $218 / 98$ & 0.119 \\
History of PONV (N/Y) & $77 / 5$ & $311 / 11$ & 0.207 \\
\hline
\end{tabular}

Abbreviations: $\mathrm{F}$, female; M, male; N, no; PONV, postoperative nausea and vomiting; $\mathrm{Y}$, yes. ${ }^{a}$ Chi-squared tests were used to perform statistical analysis of differences between the groups.

\section{Demographic data and risk factor analysis}

The collected demographic data and some reported risk factors of PON were also used to evaluate the risk effect on PON occurrence. There were no significant associations between reported PONV risk factors and PON occurrence during the 24 postoperative hours in our examined population (Table 2).

\section{Marker genotyping}

Three selected SNPs were genotyped for all our participants. The average genotyping success rate was about $94 \%$. General populationbased genotypic and allelic distributions were similar to the data from the dbSNP and international HapMap project (http://hapmap.ncbi. nlm.nih.gov/). Genotype distributions of all polymorphic markers did not deviate from Hardy-Weinberg equilibrium in any of the participants $(P=0.18-0.93)$.

\section{Initial exploratory genetic study}

The genetic association of the HTR3A polymorphisms with the PON phenotype was tested in patients who underwent operations 1 and 2, which were considered similar operations because they involved open abdomens. Three SNPs were analyzed, using the the Pearson's $\chi^{2}$ test, for differences between patients with and without the PON phenotype during the first 24 postoperative hours. All of the HTR3A gene polymorphisms were significantly associated with PON (Table 3; Supplementary Table S1), and two SNPs remained significantly associated after Bonferroni corrections (0.015 for rs33940208 and 0.024 for rs10160548).

\section{Extension association study}

To extend the association signal identified by the first-stage genetic study to more types of operations, we genotyped all three HTR3A SNPs in our extended postoperative sample of 369 patients after 9 different surgery types. The homogeneity of odds ratio were test for each group to confirm the difference of surgery types between two stages does not have a stratum-specific effect on genetic associations between HTR3A polymorphisms and PON. The homogeneity of odds ratios (ORs) were tested for each SNP, and the results showed the ORs 
Table 3 Genotypic distribution of human HTR3A gene polymorphisms

\begin{tabular}{|c|c|c|c|c|c|c|c|}
\hline \multirow[b]{3}{*}{ SNP (alleles) } & \multicolumn{5}{|c|}{ Operations 1 and 2} & \multicolumn{2}{|c|}{ All operations } \\
\hline & \multirow[b]{2}{*}{ Group (N) } & \multicolumn{4}{|c|}{ Genotype $^{\mathrm{a}}$} & \multicolumn{2}{|c|}{ Mantel-Haenszel test } \\
\hline & & 11 & 12 & 22 & P-value & P-value $e^{b}$ & Common OR $(95 \% \mathrm{Cl})^{\mathrm{c}}$ \\
\hline \multirow[t]{2}{*}{ rs33940208 (C/T) } & Nausea (44) & 34 & 9 & 1 & 0.005 & 0.002 & $0.393(0.217-0.711)$ \\
\hline & Non-nausea (127) & 63 & 51 & 13 & & & \\
\hline \multirow[t]{2}{*}{ rs1985242 (T/A) } & Nausea (41) & 22 & 13 & 6 & 0.024 & 0.004 & $2.262(1.325-3.862)$ \\
\hline & Non-nausea (123) & 37 & 59 & 27 & & & \\
\hline \multirow[t]{2}{*}{ rs10160548 (T/G) } & Nausea (36) & 20 & 12 & 4 & 0.008 & 0.012 & $0.456(0.253-0.821)$ \\
\hline & Non-nausea (116) & 32 & 64 & 20 & & & \\
\hline
\end{tabular}

Abbreviations: $\mathrm{Cl}$, confidence interval; HTR3A, 5-hydroxytryptamine type 3 A receptor; OR, odds ratio; SNP, single-nucleotide polymorphism.

a Homozygous major allele $=11$, heterozygous major allele $=12$, and homozygous minor allele $=22$.

bMantel-Haenszel chi-square statistic.

'The Mantel-Haenszel estimate of the common odds ratio.

Table 4 Pairwise linkage disequilibrium $\left(D^{\prime}\right)$ of the HTR3A SNPs in nausea and non-nausea patients

\begin{tabular}{lccc}
\hline & $r s 33940208$ & $r s 1985242$ & $r s 10160548$ \\
\hline rs33940208 & & 0.986 & 0.404 \\
rs1985242 & 1 & & 0.642 \\
rs10160548 & 0.446 & 0.725 & \\
\hline
\end{tabular}

Abbreviations: HTR3A, 5-hydroxytryptamine type 3 A receptor; SNP, single-nucleotide polymorphism.

The upper triangle is the non-nausea patients and the lower triangle shows the nausea patients.

were homogeneous across two data sets ( $P$-value from 0.115 to 0.436$)$. Therefore, we applied Mantel-Haenszel method to perform our second-stage association. The Mantel-Haenszel chi-square test for the overall degree of association showed significant associations between all of the HTR3A gene polymorphisms and PON (Table 3) and remained significantly associated after Bonferroni corrections ( $P$-value from 0.006 to 0.036$)$.

\section{Haplotype association analysis}

Because three SNPs showed consistent associations across two stages of analysis, we did pairwise LD analyses between these SNPs across the HTR3A gene in all patients. The estimated pairwise LD coefficients $\left(D^{\prime}\right)$ were used to represent the LD between the three markers (Table 4). Strong LD was detected between adjacent SNP pairs but not for separated pairs. It was of interest to investigate whether HTR3A haplotypes were also associated with PON. For the three HTR3A SNPs, haplotype construction and frequency estimation was estimated using SNPAlyze. There were five common haplotypes with a frequency $>5 \%$ in all patients who underwent operations 1 and 2 (Table 5). In addition, $P$-values estimated from 1000 permutation tests were obtained for each analysis. For the PON association, the distribution of the most common haplotype, CTT, showed a significant risk effect $(P=0.003$; OR: $2.31,95 \%$ confidence interval $(\mathrm{CI})$ : 1.34-4.01). The second common haplotype differed in non-nausea (TAG) and PON groups (CAG), and the TAG haplotype was associated with a significant protective effect $(P=0.005$; OR: $0.28,95 \%$ CI: $0.11-0.73)$. These two common haplotypes account for almost $70 \%$ of the total number of haplotypes. Haplotype analysis of the samples extended to all nine different types of operations also showing significant results for predictive haplotypes (Table 5). Furthermore, $P$-values from permutation tests for PON and predictive haplotype associations were still significant in both two-stage studies (Table 5).

\section{DISCUSSION}

We screened for HTR3A polymorphisms as genetic factors for PON susceptibility. Because ethnicity is important for identifying genetic markers, we examined the genetic variants of the HTR3A gene in postoperative patients in a unique Taiwanese population. There were no significant associations reported between PONV risk factors and PON occurrence during 24 postoperative hours in this population. We found that all three HTR3A polymorphisms were significantly associated with PON and that they could be good predictors for PON on a single-locus level. LD-based haplotypes with multiple loci are also good predictors for PON phenotypes. Two of the most common haplotypes, CTT and TAG, provided a significant risk for and a significant protective effect against, respectively, for PON.

Our results differed from those of other PONV-related genetic studies, because our study was specifically focused on investigating genetic determinants of PON. PONV is defined as any postoperative nausea, retching or vomiting, and these three distinctive phenomena have usually been grouped together as a single clinical symptom. However, the different incidences and possible risk factors suggested that, although these three phenotypes are related, they are actually unique symptoms. ${ }^{8,9,23-25}$ Mapping genetic components of each symptom independently is critical to precisely predict the occurrence of these symptoms in a given patient. Current theory also suggests that phenotypic clarity may be a promising strategy for identifying the genetic factors of a complex syndrome with a heterogeneous etiology. ${ }^{26}$ We have previously discerned the phenotype correlation between PON and POV in our study population, and a low correlation between the severity of PON and POV (Pearson's $r$ : 0.27, $P$-value $<0.001$ ) was found (unpublished data). Here we further validate reported PONV risk factors for all our participants. There were no significant associations between any proposed PONV risk factors, including gender, non-smoking, a history of motion sickness or PONV and so on, ${ }^{27}$ with PON occurrence in our examined population. These results agree with those previously proposed that PON and POV should be analyzed separately as two biologically different phenomena. ${ }^{9,25}$

On the other hand, most current studies on the effects of genetic polymorphisms are focused on POV only or on their pharmacogenetic roles: nausea- and vomiting-related drug efficacy, ${ }^{21,28-33}$ and specific anesthetics that may cause nausea, vomiting or both and so on. For 
Table 5 Haplotype frequency and test statistics between the nausea and non-nausea group patients

\begin{tabular}{|c|c|c|c|c|c|c|c|c|c|c|}
\hline \multirow[b]{3}{*}{ Haplotype $e^{\mathrm{a}, \mathrm{b}}$} & \multicolumn{5}{|c|}{ Stage One } & \multicolumn{5}{|c|}{ Stage Two } \\
\hline & \multicolumn{5}{|c|}{ Number (frequency) } & \multicolumn{5}{|c|}{ Number (frequency) } \\
\hline & Overall & Non-nausea & Nausea & $\mathrm{P}$ (permutation) & OR $(95 \% \mathrm{Cl})$ & Overall & Non-nausea & Nausea & $\mathrm{P}$ (permutation) & OR $(95 \% \mathrm{Cl})$ \\
\hline C-T-T & $151(0.50)$ & $104(0.45)$ & $47(0.65)$ & $0.003(0.001)$ & $2.31(1.34-4.01)$ & $336(0.53)$ & $264(0.45)$ & $72(0.64)$ & $0.008(0.014)$ & $1.77(1.16-2.71)$ \\
\hline T-A-G & $54(0.18)$ & $49(0.21)$ & $5(0.07)$ & $0.005(0.011)$ & $0.28(0.11-0.73)$ & $95(0.15)$ & $87(0.21)$ & $8(0.07)$ & $0.009(0.018)$ & $0.39(0.18-0.82)$ \\
\hline C-T-G & $26(0.09)$ & $22(0.09)$ & $4(0.06)$ & 0.305 (0.329) & $0.56(0.19-1.69)$ & $52(0.08)$ & $46(0.09)$ & $6(0.06)$ & $0.264(0.283)$ & $0.58(0.24-1.41)$ \\
\hline
\end{tabular}

Abbreviations: $\mathrm{Cl}$, confidence interval; $\mathrm{OR}$, odds ratio.

a Haplotypes were constructed using three single-nucleotide polymorphisms (from left to right): rs33940208, rs1985242, rs10160548.

bThe haplotypes with frequencies of $<0.05$ were not included in haplotype association analysis.

example, one study ${ }^{32}$ reported that, in patients undergoing cancer therapy, the HTR3A gene may not be a pharmacogenetic predictor for anti-emetic treatment in patients with 5-HT3 receptor antagonists, and another ${ }^{33}$ said that the variations within the HTR3B gene are predictors of the efficacy of anti-emetic treatment. The SNPs of the HTR3B, COMT and CHRM3 genes may be correlated with the variability of nausea in cancer patients taking opioids. ${ }^{29}$ However, there are still few risk factors used to predict PON, especially genetic risk factors; hence, our current efforts in the identification of predictive genetic markers for PON is worth pursuing.

Although the results from our study were significant, it is possible that we had false positives because of our relatively small sample size. Nevertheless, using a two-stage association approach should give our findings greater credibility. In our study, the statistical significance of single-locus- and haplotype-based association were consistent across the two-study stages. Moreover, permutation tests for PON and predictive haplotype association were still significant in both twostage studies. We, therefore, expected a large genetic impact of HTR3A variants in patients with $\mathrm{PON}$, which could be a useful genetic predictor. Our findings support the phenotypic dissection strategy, which hypothesizes that only one specific clinical symptom will allow us to identify the genetic components of the heterogeneous PONV. Moreover, because we analyzed patients who had undergone nine different types of surgeries and most of the known PONV risk factors could not be used to predict PON occurrence; we suggest that genetic background, especially harboring specific variants of the HTR $3 A$ gene, may be a key contributor and general predictor of PON in the unique Taiwanese population. These data are promising, and further independent validation in Taiwanese population should be performed to evaluate the generalizability of our findings.

Although two of the three polymorphisms assessed in this study are located in introns and may indirectly affect the occurrence of PON, our two-stage validation showed that they were good predictors of postsurgery PON. In addition, we cannot exclude the possibility of other variants that exist within the HTR3A gene or neighboring gene area which can also participate in the PON development. In particular, the HTR3 gene encodes the other subunit of the HTR3 receptor, HTR3B, which is located next to HTR3A and specifically modifies its function, which also serves as a major determinant for serotonin receptor 3. ${ }^{19,30,33,34}$ Investigation on the predictive value of HTR3B polymorphisms and its interaction with the HTR3A gene will provide valuable information for the pathoetiology and predictive effects of HTR3 variations associated with PON.

In conclusion, the predictive ability of single-locus- and haplotypebased HTR3A polymorphisms on PON occurrence are validated in a two-stage association study. These polymorphisms could be useful in stratifying Taiwanese patients to a risk of nausea after general anesthesiology in different kinds of operations. Significant differences were noted in haplotypes associated with both risk and protection effects, suggesting that a modulation exists in the HTR3A locus. This conflict in the effects presented in the haplotype nested in the same area can be considered as an interaction between them. The reciprocal effect may help establish a model to predict the phenotype associated with a specific haplotype, which is complicated but more realistic. Knowledge of such kinds of 'personal predictive markers' should help physicians identify patients at particular risk and help improve the management of PON.

\section{CONFLICT OF INTEREST}

The authors declare no conflict of interest.

\section{ACKNOWLEDGEMENTS}

This study was supported by grant R-98-2 from the Ditmanson Medical Foundation Chia-Yi Christian Hospital, Chia-Yi, Taiwan. We thank Hsiang-Hua Wu, MS (Research Assistant, Institute of Molecular Medicine, National Cheng Kung University, Tainan, Taiwan), for data management assistance and Bill Franke, MA, for English editorial assistance.

1 Apfel, C. C., Laara, E., Koivuranta, M., Greim, C. A. \& Roewer, N. A simplified risk score for predicting postoperative nausea and vomiting: conclusions from cross-validations between two centers. Anesthesiology 91, 693-700 (1999).

2 Cohen, M. M., Duncan, P. G., DeBoer, D. P. \& Tweed, W. A. The postoperative interview: assessing risk factors for nausea and vomiting. Anesth. Analg. 78, 7-16 (1994).

3 Watcha, M. F. Postoperative nausea and emesis. Anesthesiol. Clin. North Am. 20, 709-722 (2002)

4 Apfel, C. C., Korttila, K., Abdalla, M., Kerger, H., Turan, A., Vedder, I. et al. A factorial trial of six interventions for the prevention of postoperative nausea and vomiting. N. Engl. J. Med. 350, 2441-2451 (2004).

5 Eberhart, L. H. \& Morin, A. M. Risk scores for predicting postoperative nausea and vomiting are clinically useful tools and should be used in every patient: con-'life is really simple, but we insist on making it complicated'. Eur. J. Anaesthesiol. 28 155-159 (2011).

6 Pierre, S. Risk scores for predicting postoperative nausea and vomiting are clinically useful tools and should be used in every patient: pro-'don't throw the baby out with the bathwater'. Eur. J. Anaesthesiol. 28, 160-163 (2011).

7 Neufeld, S. M., Newburn-Cook, C. V., Schopflocher, D., Dundon, B., Yu, H. \& Drummond, J. E. Children's vomiting following posterior fossa surgery: a retrospective study. BMC Nurs, 8, 7 (2009).

8 Gan, T. J., Meyer, T. A., Apfel, C. C., Chung, F., Davis, P. J., Habib, A. S. et al. Society for Ambulatory Anesthesia guidelines for the management of postoperative nausea and vomiting. Anesth. Analg. 105, 1615-1628 (2007).

9 Stadler, M., Bardiau, F., Seidel, L., Albert, A. \& Boogaerts, J. G. Difference in risk factors for postoperative nausea and vomiting. Anesthesiology 98, 46-52 (2003). 
10 Cruthirds, D., Sims, P. J. \& Louis, P. J. Review and recommendations for the prevention, management, and treatment of postoperative and postdischarge nausea and vomiting. Oral Surg. Oral Med. Oral Pathol. Oral Radiol. 115, 601-611 (2013).

11 Becker, D. E. Nausea, vomiting, and hiccups: a review of mechanisms and treatment. Anesth. Prog. 57, 150-156; quiz 157 (2010).

12 Gan, T. J. Risk factors for postoperative nausea and vomiting. Anesth. Analg. 102, 1884-1898 (2006)

13 Eberhart, L. H., Geldner, G., Kranke, P., Morin, A. M., Schauffelen, A., Treiber, H. et al. The development and validation of a risk score to predict the probability of postoperative vomiting in pediatric patients. Anesth. Analg. 99, 1630-1637 (2004).

14 Tyers, M. B. \& Freeman, A. J. Mechanism of the anti-emetic activity of 5-HT3 receptor antagonists. Oncology 49, 263-268 (1992).

15 Ueda, N., Yoshimura, R., Shinkai, K., Sakata, Y. \& Nakamura, J. Higher plasma 5-hydroxyindoleacetic acid levels are associated with SSRI-induced nausea. Neuropsychobiology 48, 31-34 (2003).

16 Ho, K. Y. \& Gan, T. J. Pharmacology, pharmacogenetics, and clinical efficacy of 5hydroxytryptamine type 3 receptor antagonists for postoperative nausea and vomiting. Curr. Opin. Anaesthesiol. 19, 606-611 (2006)

17 Tang, D. H. \& Malone, D. C. A network meta-analysis on the efficacy of serotonin type 3 receptor antagonists used in adults during the first $24 \mathrm{~h}$ for postoperative nausea and vomiting prophylaxis. Clin. Ther. 34, 282-294 (2012).

18 Boyd, G. W., Low, P., Dunlop, J. I., Robertson, L. A., Vardy, A., Lambert, J. J. et al. Assembly and cell surface expression of homomeric and heteromeric 5-HT3 receptors: the role of oligomerization and chaperone proteins. Mol. Cell. Neurosci. 21, 38-50 (2002).

19 Dubin, A. E., Huvar, R., D'Andrea, M. R., Pyati, J., Zhu, J. Y., Joy, K. C. et al. The pharmacological and functional characteristics of the serotonin 5-HT(3A) receptor are specifically modified by a 5-HT(3B) receptor subunit. J. Biol. Chem. 274, 30799-30810 (1999).

20 Watcha, M. F. \& White, P. F. Postoperative nausea and vomiting. its etiology, treatment, and prevention. Anesthesiology 77, 162-184 (1992).

21 Singhal, A. K., Kannan, S. \& Gota, V. S. 5HT3 antagonists for prophylaxis of postoperative nausea and vomiting in breast surgery: a meta-analysis. J. Postgrad. Med. 58, 23-31 (2012).

22 de Bakker, P. I., Yelensky, R., Pe'er, I., Gabriel, S. B., Daly, M. J. \& Altshuler, D. Efficiency and power in genetic association studies. Nat. Genet. 37, 1217-1223 (2005).
23 Rhodes, V. A. \& McDaniel, R. W. Nausea, vomiting, and retching: complex problems in palliative care. CA Cancer J. Clin. 51, 232-248; quiz 249-252 (2001).

24 Wood, J. M., Chapman, K. \& Eilers, J. Tools for assessing nausea, vomiting, and retching. Cancer Nurs. 34, E14-E24 (2011).

25 Tramer, M. R. A rational approach to the control of postoperative nausea and vomiting evidence from systematic reviews. Part II. Recommendations for prevention and treatment, and research agenda. Acta Anaesthesiol. Scand. 45, 14-19 (2001).

26 Risch, N. J. Searching for genetic determinants in the new millennium. Nature 405, 847-856 (2000).

27 Jokinen, J., Smith, A. F., Roewer, N., Eberhart, L. H. \& Kranke, P. Management of postoperative nausea and vomiting: how to deal with refractory PONV. Anesthesiol. Clin. 30, 481-493 (2012).

28 Tanaka, M., Kobayashi, D., Murakami, Y., Ozaki, N., Suzuki, T., Iwata, N. et al. Genetic polymorphisms in the 5-hydroxytryptamine type $3 \mathrm{~B}$ receptor gene and paroxetineinduced nausea. Int. J. Neuropsychopharmacol. 11, 261-267 (2008).

29 Laugsand, E. A., Fladvad, T., Skorpen, F., Maltoni, M., Kaasa, S., Fayers, P. et al. Clinical and genetic factors associated with nausea and vomiting in cancer patients receiving opioids. Eur. J. Cancer 47, 1682-1691 (2011).

30 Rueffert, H., Thieme, V., Wallenborn, J., Lemnitz, N., Bergmann, A., Rudlof, K. et al Do variations in the 5-HT3A and 5-HT3B serotonin receptor genes (HTR3A and HTR3B) influence the occurrence of postoperative vomiting? Anesth. Analg. 109, 1442-1447 (2009).

31 Sugai, T., Suzuki, Y., Sawamura, K., Fukui, N., Inoue, Y. \& Someya, T. The effect of 5-hydroxytryptamine $3 \mathrm{~A}$ and $3 \mathrm{~B}$ receptor genes on nausea induced by paroxetine. Pharmacogenomics J. 6, 351-356 (2006).

32 Kaiser, R., Tremblay, P. B., Sezer, O., Possinger, K., Roots, I. \& Brockmoller, J. Investigation of the association between 5 - HT3A receptor gene polymorphisms and efficiency of antiemetic treatment with 5 -HT3 receptor antagonists. Pharmacogenetics 14, 271-278 (2004).

33 Tremblay, P. B., Kaiser, R., Sezer, O., Rosler, N., Schelenz, C., Possinger, K. et al. Variations in the 5-hydroxytryptamine type 3B receptor gene as predictors of the efficacy of antiemetic treatment in cancer patients. J. Clin. Oncol. 21, 2147-2155 (2003).

34 Davies, P. A., Pistis, M., Hanna, M. C., Peters, J. A., Lambert, J. J., Hales, T. G. et al. The 5-HT3B subunit is a major determinant of serotonin-receptor function. Nature 397 359-363 (1999).

Supplementary Information accompanies the paper on Journal of Human Genetics website (http://www.nature.com/jhg) 